\title{
SPECIAL DIVISORS ON COMPACT RIEMANN SURFACES ${ }^{1}$
}

\author{
HERSHEL M. FARKAS
}

Introduction. In a previous paper [1] the author obtained some results concerning the distribution of special divisors on compact Riemann surfaces of genus $g$. The reader is referred to [1] for definitions and notation.

It was shown in [1, p. 886] that the product structure with $S_{r} \times S_{r}$ and $T^{\circ}(S)$ as factors may be endowed with a structure of complex analytic manifold in such a way that the resulting space, $W_{r, r}$, is an analytic fibre space over the base manifold $T^{o}(S)$ with fibre $S_{r} \times S_{r}$ over $S(T) \in T^{\circ}(S)$. We then proved [1, Theorem 2] that if $\zeta, \omega$ are completely distinct equivalent special divisors of degree $r$ on $S_{0}$, then considering the triple $\left(\zeta, \omega, S_{0}(T)\right)$ as a point in $W_{r, r}$, there is a $g$ codimensional submanifold of $W_{r, r}$ containing the point $\left(\zeta, \omega, S_{0}(T)\right)$ (each point of which has projections onto pairs of equivalent, special divisors on the surface $S_{0}(T)$, the base point under the fibre) which projects onto a $\lambda$ codimensional submanifold of $T^{o}(S)$. Bounds were obtained for $\lambda$, and from these bounds it followed that a special divisor of degree $r<(g+1) / 2$ is always special in the sense of moduli. Finally we showed that if $g$ is odd, a special divisor of degree $(g+1) / 2$ is also special in the sense of moduli. Hence our results for special divisors were [1, Theorem 5] that if $g$ is even (odd), a special divisor of degree less than $(g+2) / 2((g+3) / 2)$ is special in the sense of moduli. As a particular example of the method, we computed the dimension of the sublocus of $T^{\circ}(S)$ possessing Weierstrass points whose Weierstrass sequences begin with a fixed $r<g$.

It is the purpose of this note to indicate that the techniques used in [1] can be employed to yield a general theorem from which the results of [1] emerge as corollaries. Furthermore, in our present treatment, we shall obtain directly that a special divisor of degree less than $(g+2) / 2$ is special in the sense of moduli, eliminating the necessity of Theorem 4 in [1].

1. Suppose $f$ is a meromorphic function with $r$ zeros and $r$ poles on $S$ a compact Riemann surface of genus $g$. Then, by Abel's theorem, $S$ possesses a pair of integral, equivalent, completely distinct, special divisors of degree $r$, and $f$ projects $S$ on to an $r$-sheeted branched cover

Received by the editors January 13, 1967.

1 The research was partially supported by the NFS GP-3452. 
of the Riemann sphere with $2 r+2 g-2$ branch points. Conversely, if $S$ permits a representation as an $r$-sheeted cover of the sphere, $S$ possesses a pair of equivalent, integral, completely distinct, special divisors of degree $r$. Hence we see that the existence of a pair of integral, equivalent, completely distinct, special divisors on $S$ is equivalent to the existence of an $r$-sheeted concrete representation of the surface $S$. With this in mind, the results of [1] mentioned in the introduction can be restated in the following way: for $g$ even (odd) the property of permitting an $r$-sheeted representation is special in the sense of moduli if $r<(g+2) / 2((g+3) / 2)$.

Having stated the results in this form, the following question naturally presents itself. Let $S$ be a compact Riemann surface of genus $g$ which permits an $r$-sheeted concrete representation with an $(s-1)$ th order branch point $2 \leqq s \leqq r$. When is such a representation special in the sense of moduli? Before we answer this question, we observe that the desired property is equivalent to the demand that $S$ possess a pair of integral, equivalent, special divisors $\zeta=P^{8} P_{1} \ldots$ $P_{r-s}, \omega=Q_{1} \cdots Q_{r}$. This follows by virtue of the fact that we can always arrange that the branch point of order $s-1$ lie over the origin or $\infty$.

We recall that an integral divisor $\zeta$ of degree $r<g$ is said to be special if $i(\zeta)=g-r+1+m, m \geqq 0$.

THEOREM 1. The dimension $d$ of the locus of $T^{\circ}(S)$ whose underlying surfaces permit $r$-sheeted representations with an $(s-1)$ th order branch point satisfies the inequalities

$$
r+2 g-4-m \leqq d \leqq \min (V-m-(s+1), 3 g-3)
$$

where $V=2 r+2 g-2$ and $m$ is computed from the index of specialty of the divisor.

Proof. As we have already indicated, the desired property is equivalent to the existence on $S$ of integral, equivalent, completely distinct, special divisors $\zeta=P^{s} P_{1} \cdots P_{r-s}, \omega=Q_{1} \cdots Q_{r}$.

By Abel's theorem

$$
u_{i}(\zeta)-u_{i}(\omega)-m_{i}-\sum_{j=1}^{g} n_{j} \Pi_{i j}=0, \quad i=1, \cdots g,
$$

where $m_{i}, n_{j}$ are integers. These equations are of course the same as

$$
\begin{aligned}
s u_{i}(P) & +u_{i}\left(P_{1}\right)+\cdots+u_{i}\left(P_{r-s}\right)-u_{i}\left(Q_{1}\right)-\cdots-u_{i}\left(Q_{r}\right)-m_{i} \\
& -\sum_{j=1}^{0} n_{j} \Pi_{i j}=0 .
\end{aligned}
$$


We now view these equations as ranging over $W_{r-s+1, r}$, and just as in the proof of Theorem 2 in [1], define therein a $g$ codimensional submanifold, each point of which has projections onto pairs of equivalent, integral, special divisors of the specified form which projects onto a $\lambda$ codimensional submanifold of $T^{\circ}(S)$. The same sort of estimates which yielded Theorem 3 in [1] give here that

$$
g-2 r+m+s \leqq \lambda \leqq g-r+1+m
$$

and we therefore immediately obtain the desired inequalities on $d$.

We now restrict ourself to the case $m=0$. If $m>0$, then $S$ permits a concrete representation with fewer than $r$ sheets. If $m=0$ the inequalities on $d$ are

$$
r+2 g-4 \leqq d \leqq \min (V-(s+1), 3 g-3) .
$$

COROLLARY Let $S$ be a compact Riemann surface of genus g. The property of $S$ permitting an $r$-sheeted concrete representation with an $(s-1)$ th order branch point is special in the sense of moduli if $r<(g+s) / 2$.

Proof. By definition, the property is special if $d<3 g-3$. $d<3 g-3$ if $r<(g+s) / 2$.

2. In [1] we considered arbitrary, integral, special divisors of degree $r<g$. These special divisors of course give rise to meromorphic functions which project the Riemann surface onto a concrete $r$ sheeted cover of the sphere with $2 r+2 g-2$ branch points. The point is, and this is what we omitted mentioning in [1], that even if the branch points are all simple we can still assume that the special divisor is of the form $P^{2} P_{1} \cdots P_{r-2}$. Hence, we can apply our theorem with $s=2$ and obtain the result that a special divisor of degree $<(g+2) / 2$ is always special in the sense of moduli, and we do not have to appeal to Theorem 4 of [1].

In the case of a Weierstrass point whose Weierstrass sequence begins with a fixed $r<g$, we have $s=r$ and hence the property is special if $r<(g+r) / 2$ or if $r<g$. Furthermore, in this case, the inequalities satisfied by $d$ are

$$
r+2 g-4 \leqq d \leqq r+2 g-3 .
$$

Returning for a moment to the case $s=2$ and $m=0$, we see that in this case the inequalities on $d$ are

$$
r+2 g-4 \leqq d \leqq \min (V-3,3 g-3) .
$$

Hence, we see that if there are few enough branch points, we obtain the result of Riemann that after normalizing 3 branch points the 
number that remain are an upper bound for the dimension of the space of moduli. In connection with these remarks the reader is referred to [2] for a more complete discussion.

The author is indebted to Professor H. E. Rauch for many interesting and stimulating discussions concerning the research reported on in this note.

\section{REFERENCES}

1. H. M. Farkas, Special divisors and analytic subloci of Teichmueller space, Amer. J. Math. 88 (1966), 881-901.

2. H. E. Rauch, Weierstrass points, branch points and moduli of Riemann surfaces, Comm. Pure Appl. Math. 12 (1959), 543-560.

Johns Hopkins UNiversity 